\title{
Paging Strategies for Highly Mobile Users
}

\author{
Aylin Yener \\ Christopher Rose \\ Wireless Information Networks Laboratory (WINLAB) \\ Department of Electrical and Computer Engineering \\ Rutgers University \\ PO Box 909 Piscataway NJ 08855-0909 \\ yener@winlab.rutgers.edu, crose@winlab.rutgers.edu
}

\begin{abstract}
In this work, we consider the problem of minimizing average paging cost and polling delay in a wireless system. It is assumed that users to be paged move according to a general motion process during the paging events. We find and evaluate the optimal sequential paging strategies for given maximum delay tolerance and compare our results to the classical paging strategy as well as the optimal strategies for stationary units (users).
\end{abstract}

\section{Introduction}

In mobile communications systems, timely localization of users is an important factor in service quality. Since the excess signaling imposed by user mobility can be excessive $[1,2]$ and radio bandwidth is scarce in wireless systems, paging algorithms that minimize signaling are desirable.

Previous work [3-5] has considered optimal paging strategies when the mobile unit is assurned to remain essentially stationary for the duration of the paging process. However, if paging bandwidth is scarce, or the system is heavily loaded, this assumption may be inappropriate. Thus, we here consider optimal strategies to locate a mobile unit when the unit may change location between the polling events; i.e., it might be necessary to poll a given location multiple times rather than just once as is in the stationary case.

In the following sections, we first formulate the problem of finding the optimal paging strategies. We then present the results obtained under different assumptions and the conclusions of our work.

\section{Basics}

For a given unit, groups of locations $A_{n}$ are polled in sequence, $n=1,2 \ldots N$ until the unit is found. The probability of finding the unit on the $n^{\text {th }}$ polling step is defined as $q_{n}$. The number of locations in each group is $k_{n}$. If the unit is found on the $n^{\text {th }}$ step then the total number of locations polled to that point is

$$
s_{n}=\sum_{i=1}^{n} k_{i}
$$

Using the number of locations searched as a surrogate for signaling cost we have

$$
E[P]=\sum_{n=1}^{N} q_{n} s_{n}=\sum_{n=1}^{N} k_{n} \bar{F}_{q}(n-1)
$$

and as a surrogate for polling delay we have

$$
E[D]=\sum_{n=1}^{N} n q_{n}=\sum_{n=1}^{N} \tilde{F}_{q}(n-1)
$$

the mean number of polling events. Note that $\tilde{F}_{q}(n)$ is the complementary cumulative distribution function of $q_{n}$ or formally

$$
\bar{F}_{q}(n)=1-\sum_{i=1}^{n} q_{i}
$$

Similar to [3], we seek to minimize the weighted sum (weighting factor $\alpha \geq 0$ ) of $E[P]$ and $E[D]$,

$$
G=\sum_{n=1}^{N}\left(s_{n}+\alpha n\right) q_{n}=\sum_{n=1}^{N} \bar{F}_{q}(n-1)\left(k_{n}+\alpha\right)
$$

by optimally choosing both the size of polling groups $k_{n}$ and their elements $i \in A_{n}, i=1,2 \ldots k_{n}$.

We assume that the unit to be paged moves according to a general motion process. The joint probability distribution on unit location at time $t_{n}$ can be written in terms of conditional probabilities as: ${ }^{1}$

$$
p_{X_{1} \ldots X_{n}}\left(x_{1} \ldots x_{n}\right)=\prod_{i=1}^{n} p_{X_{i} \mid X_{i-1} \ldots X_{1}}\left(x_{i} \mid x_{i-1} \ldots x_{1}\right)
$$

\footnotetext{
${ }^{1}$ Although both time and space invariance seem to be implied by the notation, the results to be derived are applicable to the time/space varying cases. Notational simplicity was chosen over exactness.
} 
Given polling groups $A_{i}, i=1,2, \ldots$ we can write down the probability of finding the user in the $n^{\text {th }}$ step as [6]:

$$
q_{n}=\sum_{x_{n} \in A_{n}} \sum_{x_{n-1} \notin A_{n-1}} \ldots \sum_{x_{1} \notin A_{1}} p_{X_{1} \ldots X_{n}}\left(x_{1} \ldots x_{n}\right)
$$

It is easy to see that $\bar{F}_{q}(n)$ can be written as [6]:

$$
\bar{F}_{q}(n)=\bar{F}_{q}(n-1) \sum_{x_{n} \notin A_{n}} p_{X_{n} \mid \bar{B}_{n-1} . . \bar{B}_{1}}\left(x_{n} \mid \bar{B}_{n-1} . . \bar{B}_{1}\right)
$$

where $\bar{B}_{i}$ is the event that the unit is not found by polling step $i$.

We then state the following theorem:

Theorem: The polling sequence which minimizes Equation (2), (3) or (5) polls the most probable $k_{i+1}$ locations after the $i^{\text {th }}$ polling failure.

Proof: If polling groups $A_{1} \ldots A_{N-1}$ are given, then $\bar{F}_{q}(i)$ is specified for $i=1,2, \ldots, N-1$. Given $k_{N}$, minimizing any of the cost functions (2) through (5) requires us to choose the elements of $A_{N}$ such that $\vec{F}_{q}(N)$ is minimized. However, $\bar{F}_{q}(N)$ is minimized by choosing the most likely elements of $p_{X_{N} \mid \bar{B}_{n-1}}\left(x_{N} \mid \bar{B}_{N-1} \ldots \bar{B}_{1}\right)$ for $A_{N}$.

Proceeding recursively, we see that for given polling group sizes $k_{n}$, the optimal polling group $A_{n}$ contains the $k_{n}$ most likely elements of the distribution $p_{X_{n} \mid \widetilde{B}_{n-1} \ldots \bar{B}_{1}}\left(x_{n} \mid \bar{B}_{n-1} \ldots \bar{B}_{1}\right)$. Thus, the problem of finding $N$ optimal polling groups reduces to one of finding optimal polling group sizes $k_{n}$.

This result greatly simplifies the problem of finding the optimal strategies since now only optimal polling group sizes $k_{n}$ need be found. Unfortunately it is easily shown that this problem is not amenable to solution via Dynamic Programming (DP) [7] as it was for the semi-stationary unit case [3]. We therefore employ exhaustive search to find the best set of polling group sizes $\left\{k_{n}\right\}$.

\section{Results}

We show the results of several experiments performed to find the best polling strategy in the presence of a maximum delay constraint, i.e. if the mobile is not found in the first $N-1$ polling steps then all locations are polled in step $N$. A network of 20 locations is assumed with an initially uniform distribution on unit location probability.

As a check against previous work [3], we have first performed the exhaustive search for the case where the unit to be found remains stationary during the paging process. Using $\alpha=0.5$ in Equation (5), we compare previous results with the result of our search in Figure 1 and find them in complete agreement.

We then consider simple linear diffusion on an annulus (a "racetrack" model) and show how the paging strategy

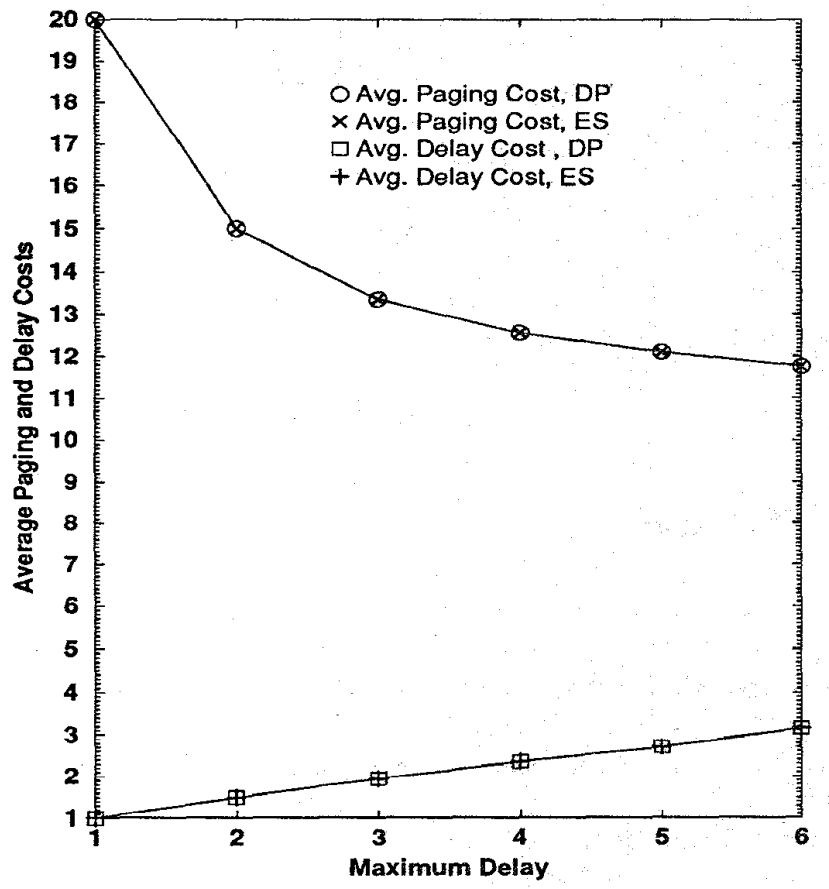

Figure 1: Optimum paging and delay costs corresponding to the optimum strategy with $\alpha=0.5$ vs maximum delay for a stationary unit, 20 locations, uniform initial location distribution. DP=Dynamic Programming, $\mathrm{ES}=$ Exhaustive Search.

varies as a function mobility index, maximum delay tolerance and delay weighting factor.

In this model, a unit moves according to a discrete isotropic diffusion process, with probability transition matrix $\mathbf{S}=\left\{s_{i j}\right\}=\left\{s_{X_{n} \mid X_{n-1}}\left(x_{n}=i \mid x_{n-1}=j\right)\right\}$, on $\mathbf{a}$ circular track with $L$ locations. Specifically,

$$
s_{i j}= \begin{cases}\gamma & \text { if } i=\bmod _{L}(j-1) \text { or } \bmod _{L}(j+1) \\ 1-2 \gamma & \text { if } j=i \\ 0 & \text { else }\end{cases}
$$

where $\gamma$ is chosen to be 0.05 . That is, the unit may move to the right or left with probability 0.05 , or remain stationary with probability 0.9 at each discrete time instant. The number of motion process steps between polling events is defined as $k(k \geq 1)$. The conditional probabilities ${ }^{2}$ then become:

$$
\begin{gathered}
\mathbf{p}_{X_{n+1} \mid \bar{B}_{n-1} . . \bar{B}_{1}}\left(x_{n+1} \mid \bar{B}_{n-1} . . \bar{B}_{1}\right)= \\
\mathbf{S}^{k} \mathbf{p}_{X_{n} \mid \bar{B}_{n-1} . . \bar{B}_{1}}\left(x_{n} \mid \bar{B}_{n-1} . . \bar{B}_{1}\right)
\end{gathered}
$$

As such, $k$ (the mobility index) is a surrogate for unit mobility $[8,9]$ with increased $k$ implying higher mobility. For $k$ sufficiently large, the probability distribution on location is uniform just prior to a polling event.

\footnotetext{
${ }^{2} \mathrm{p}$ is the $L$-vector that contains the conditional probabilities.
} 
It is observed that as the mobility index increases, the number of locations polled in each step tends to grow. The average paging cost and the average delay corresponding to the best strategies found for various mobility indices are given in Figure 2. The corresponding strategies for these and larger mobility indices with maximum delay tolerance of 6 , are given in Table 1 . Notice that the optimum strategy approaches the classical "blanket polling" strategy where all locations are polled simultaneously in the first step for very large mobility indices i.e., there is nothing to be gained by polling smaller groups since the location probability distribution is uniform at each polling step. For smaller interpolling intervals, we observe a characteristic decrease then increase in the number of locations polled at each step. This is as expected since small interpolling intervals imply that a polling failure in group $A_{i}$ greatly reduces the immediately subsequent probability of finding the unit at any location covered by $A_{i}$. However, as the number of polling failures increases, more locations are searched to avoid the penalty of searching all locations in the final step.
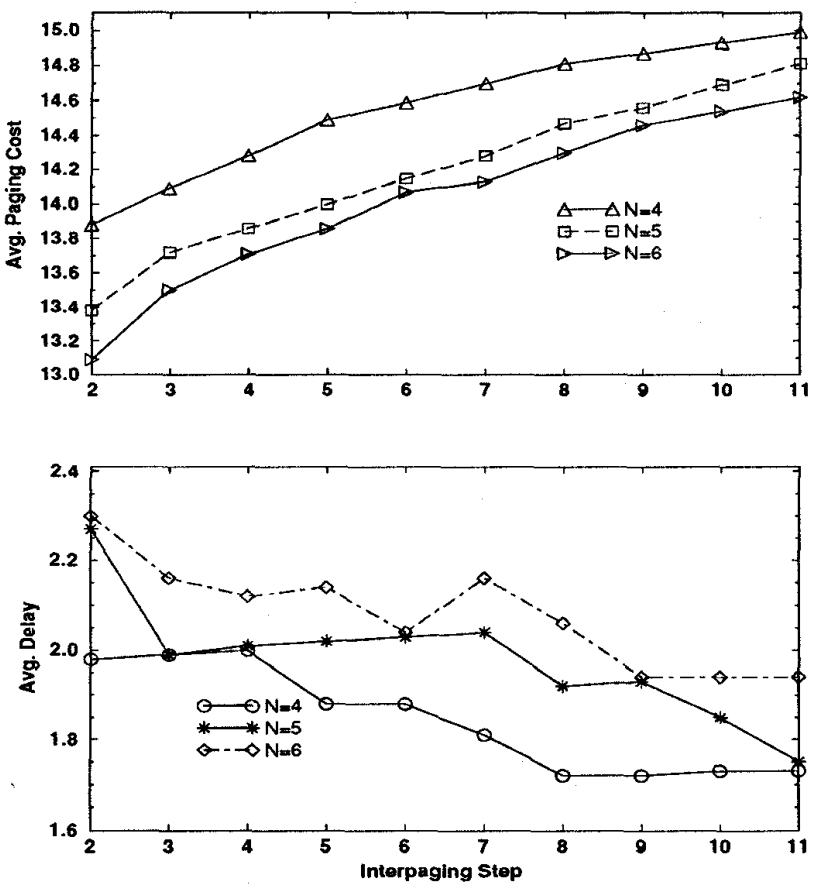

Figure 2: Paging and delay costs corresponding to the optimum total cost with $\alpha=0.5$ versus interpolling interval, 20 locations, uniform initial location distribution, race track location model.

The variation of paging cost and delay with maximum delay tolerance $N$ is also examined. What we find is a tradeoff similar to that seen in [3] where paging cost falls off sharply as delay requirements are relaxed (Figure 3 ).

Finally, we have studied the effect of delay factor $\alpha$ on the cost and the optimal strategies. The values of $E[P]$ and $E[D]$ are given in Figure 4. The values show that the pag-

\begin{tabular}{|c|c|c|c|c|c|c||}
\hline \hline I I & St.1 & St.2 & St.3 & St.4 & St.5 & St.6 \\
\hline 2 & 6 & 6 & 5 & 3 & 7 & 20 \\
\hline 3 & 7 & 6 & 5 & 4 & 7 & 20 \\
\hline 4 & 7 & 6 & 6 & 4 & 8 & 20 \\
\hline 5 & 7 & 6 & 6 & 4 & 8 & 20 \\
\hline 6 & 7 & 7 & 6 & 6 & 8 & 20 \\
\hline 7 & 7 & 6 & 6 & 5 & 9 & 20 \\
\hline 8 & 8 & 7 & 3 & 4 & 9 & 20 \\
\hline 9 & 8 & 7 & 6 & 8 & 8 & 20 \\
\hline 10 & 8 & 7 & 6 & 8 & 9 & 20 \\
\hline 11 & 8 & 7 & 6 & 9 & 11 & 20 \\
\hline 101 & 8 & 10 & 10 & 11 & 10 & 20 \\
\hline 251 & 8 & 10 & 10 & 10 & 10 & 20 \\
\hline 401 & 10 & 10 & 10 & 11 & 11 & 20 \\
\hline 451 & 11 & 11 & 11 & 11 & 11 & 20 \\
\hline 501 & 12 & 10 & 11 & 11 & 11 & 20 \\
\hline 601 & 16 & 12 & 12 & 12 & 12 & 20 \\
\hline 651 & 20 & 0 & 0 & 0 & 0 & 0 \\
\hline 701 & 20 & 0 & 0 & 0 & 0 & 0 \\
\hline 801 & 20 & 0 & 0 & 0 & 0 & 0 \\
\hline \hline
\end{tabular}

Table 1: Best polling strategies for maximum delay $=6$. The table shows how many locations should be polled at each time step for various interpolling interval values. II $=$ Interpolling Interval

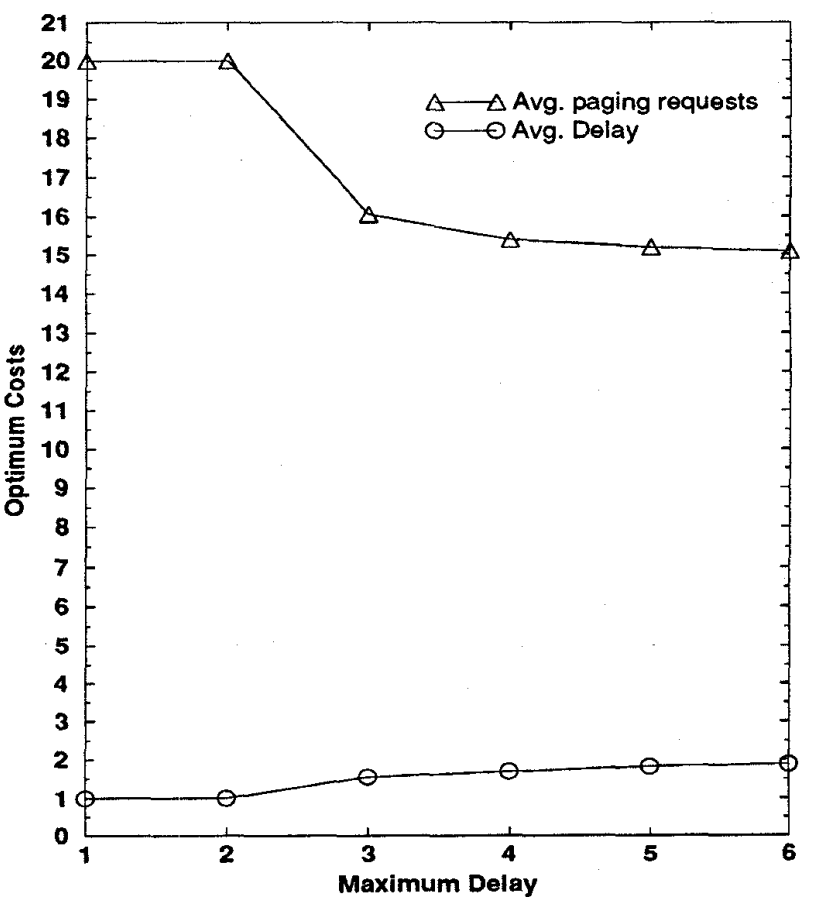

Figure 3: Optimum paging cost and the corresponding average delay versus maximum delay, $\alpha=0.5, k=20,20$ locations, uniform initial location distribution, race track location model. 


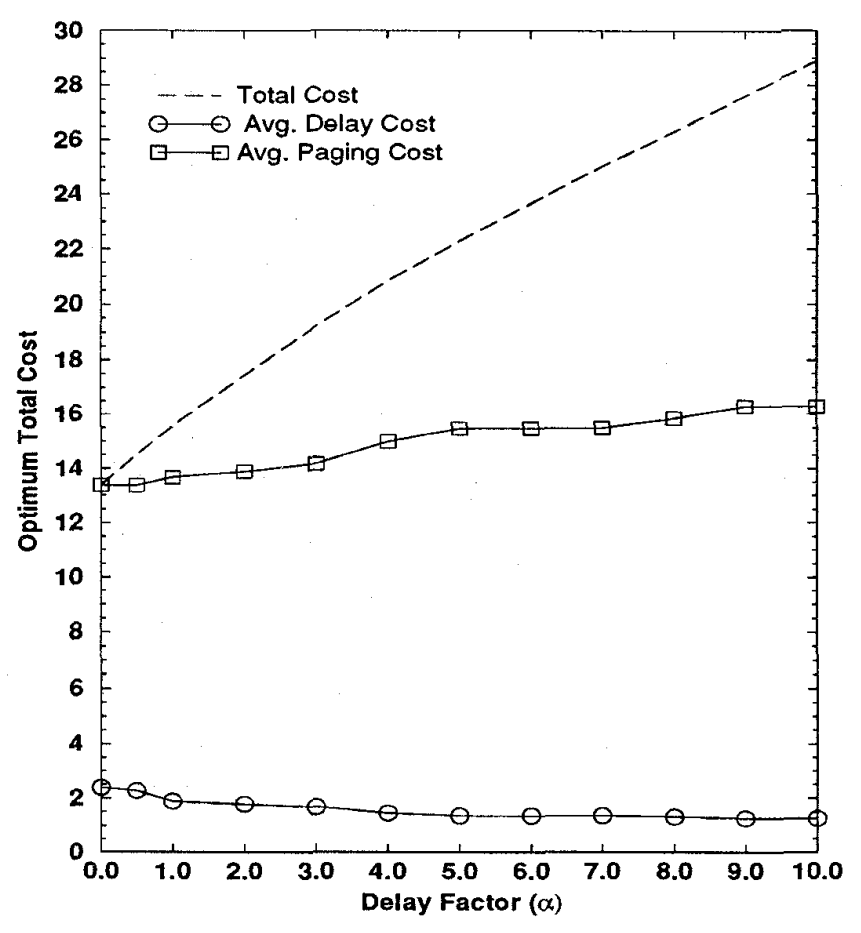

Figure 4: Optimum costs versus delay factor $\alpha$ for delay tolerance 5, 20 locations, uniform initial location distribution, race track location model.

ing cost is lowered substantially with very little increase in the average delay compared to the classical polling strategy. The optimal strategies found show that for increasing $\alpha$, a premium is placed on finding the unit early during the paging process. Thus, the size of the first group increases monotonically with $\alpha$ until all locations are searched on the first step. This strategy achieves an absolute minimum delay of one polling step.

\section{Conclusion}

We have investigated optimum paging strategies for a mobile communications network where units are allowed to move during the paging process. First, we have reduced the problem of finding the best strategies to finding how many conditionally most likely locations must be included in each polling group. The problem of finding the optimal polling group sizes is, unfortunately, not amenable to standard methods such as Dynamic Programming. Thus, we have used exhaustive search to determine the best strategy and derive heuristic/approximate principles from experiment.

We observed that as the unit mobility (time interval between polling events) increases, the number of locations searched early-on in the paging process must increase until it becomes optimal to poll all locations during the first step; the classical "blanket polling" strategy for mobile communications systems. However, this effect occurs only at relatively extreme levels of mobility where a failed polling event provides little information about unit location just prior to the next scheduled polling event. Thus, there should usually be some benefit to sequential planned polling of location groups in all but the most extreme cases.

The effect of increasing the importance of delay reduction had the expected effect of lowering delay at the expense of increased paging cost. However, the optimal strategies lowered the paging cost substantially as compared to the classical strategy but with little increase in the average delay. Thus, it may often be more efficient to sacrifice a small amount of delay performance and thereby gain a substantial reduction in paging cost.

\section{References}

[1] K.S. Meier-Hellstern, G.P. Pollini, and D.J. Goodman. Network protocols for the cellular packet switch. IEEE Transactions on Communications, 42(24/2):1235, 1994.

[2] K.S. Meier-Hellstern, E. Alonso, and D. O'Neil. The use of SS7 and GSM to support high density personal communications. In Proceedings of the International Conference on Communications ICC'92, 1992.

[3] C. Rose and R. Yates. Minimizing the average cost of paging under delay constraints. ACM Wireless Networks, 1(2):211-219, 1995.

[4] C. Rose and R. Yates. Paging Cost Minimization Under Delay Constraints. IEEE Infocom'95, April 1995. Boston, MA.

[5] S. Madhavapeddy, K. Basu, and A. Roberts. Adaptive paging algorithms for cellular systems. Fifth WINLAB Workshop on Third Generation Wireless Information Networks, April 1995. New Brunswick, NJ.

[6] C. Rose and A. Yener. Highly Mobile Users and Paging : optimal polling strategies. Submitted to IEEE Transactions on Vehicular Technology, 1995.

[7] D.P. Bertsekas. Dynamic Programming. Prentice Hall, Englewood Cliffs, NJ, 1987.

[8] C. Rose. Minimizing the average cost of paging and registration: A timer-based method. ACM Wireless Networks, 1996. (to appear).

[9] C. Rose. Minimization of paging and registration costs through registration deadlines. IEEE/ICC'95, pages 735-739, 1995. Seattle. 